\title{
Competencia territorial: un marco analítico para su estudio
}

\section{Regional competence: an analytical framework for its study}

\author{
Cristina Isabel Ibarra-Armenta* \\ Alejandra Berenice Trejo-Nieto**
}

\begin{abstract}
Within the current economic scenario of growing globalization, which goes along with a relative increase of factor mobility, the role of territories and their tendency to compete for economic success is under debate. In this context it seems relevant to question what motivates this competence, which the mechanisms behind it are, which actors participate, what its objectives and its consequences are. To answer these inquiries, based on a selective literature review, here we suggest a logic structure subject to empirical applicability to analyze what we call territorial competence. This is conceptualized as a process intrinsically linked to a phenomenon of considerable scale and scope in the literature, namely: competitiveness.
\end{abstract}

Keywords: Competitiveness, regional competence, regional disparities.

\section{Resumen}

En el actual escenario económico dominado por una creciente globalización, la cual ha estado acompańada por un incremento relativo en la movilidad de los factores productivos, se ha puesto a debate el papel de los territorios y, particularmente, su necesidad de competir por éxito económico. En este contexto es relevante cuestionar qué motiva a que las regiones o ciudades compitan entre sí, cuáles son los mecanismos de competencia, qué actores participan, cuáles son los objetivos y sus consecuencias. Para dar respuesta a estas interrogantes, con base en una selectiva revisión de literatura, aquí se propone un esquema lógico para la sistematización del análisis de lo que es denominado competencia territorial, la cual es concebida como un proceso intrínsecamente ligado a un concepto de espectro y alcance bastante amplio en la literatura conocido como competitividad.

Palabras clave: Competitividad, competencia territorial, disparidades regionales.

\footnotetext{
* Universidad Autónoma de Coahuila. Correo-e: c.ibarra-armenta.1@research.gla.ac.uk

** El Colegio de México. Correo-e: aliber23@hotmail.com
} 


\section{Introducción}

Los diferentes escenarios territoriales que sirven como puntos de articulación de las distintas relaciones económicas, sociales y políticas enfrentan constantes desafíos y oportunidades ante circunstancias dinámicas y complejas. La globalización, por ejemplo, a través de distintas vías y mecanismos, ha conducido a una creciente necesidad de numerosos territorios por aumentar su atracción y retención de recursos e inversión, que eventualmente se traduzcan en crecimiento económico y desarrollo. Esta dinámica global ha exacerbado una polarización de la que resultan espacios ganadores y perdedores. Las regiones consideradas ganadoras se caracterizan primordialmente por atraer y retener inversión productiva -generalmente en actividades generadoras de empleo y valor agregadotener altas y sostenidas tasas de crecimiento, generar excedentes exportables, etcétera. Se dice entonces que si una nación, región o territorio puede lograr esto, entonces es competitivo.

El origen de este buen desempeño territorial se ha ligado a la capacidad de adaptarse o superar una serie de condicionantes impuestas, tanto por los procesos globalizadores como por otras fuerzas propias del territorio. Esto despierta el interés y la preocupación de los gobiernos, así como de instituciones privadas y públicas y grupos de la sociedad, por proveer las condiciones que hagan atractivos a sus países, regiones o ciudades. Frecuentemente, cuando se habla de territorios competitivos se alude también a territorios con niveles altos y crecientes de productividad y calidad de vida elevados. Es así que en la práctica, los territorios han buscado fomentar aquellos factores que teóricamente contribuyen a mejorar cada uno de estos dos aspectos. No obstante, aunque de forma natural todas las regiones busquen incrementar la productividad de su base económica y la calidad de vida de sus habitantes, algunas avanzan de manera más rápida. La liberalización comercial y de inversión, la integración de bloques regionales y la globalización económica en general, han intensificado lo que parece ser una competencia auténtica entre ciudades y regiones en el marco de las economías nacionales y a nivel global (Funck, 2000; Poot, 2000; Budd, 1998). Es así como se pone en perspectiva la existencia de esta disputa entre territorios para enmarcar el concepto de competencia territorial.

A pesar de la evidente importancia de estos procesos de disputa entre territorios derivados de la dinámica global y del amplio debate acerca de la competitividad, hasta ahora no se ha desarrollado un esquema de análisis de competencia territorial como tal y su análisis empírico sistemático ha sido poco consistente (Batey y Friedrich, 2000). Este último requiere, al menos, de una estructura lógica que permita ubicar en pers- 
pectiva a la competencia territorial en relación con la competitividad y, sistematizar el estudio de la primera, de manera que pueda aplicarse a casos empíricos diversos. El objetivo de esta investigación es proponer un marco analítico integrando los aportes de diversos trabajos teóricos y empíricos dirigidos al análisis de la competencia territorial. La principal motivación se deriva del predominio de literatura acerca de la competitividad que se centra en comparaciones relativas, basadas en rankings entre regiones y que no abordan ni evidencian una lógica de competencia territorial. En los siguientes apartados se presenta tal propuesta partiendo, en primer lugar, de una revisión del concepto de competitividad y sus determinantes. Posteriormente, se hace una aproximación al término competencia territorial y se ubica en relación con el de competitividad. Después se enumeran los elementos que en consenso resultan más útiles para caracterizar a la competencia territorial, lo cual sirve de base para proponer el esquema de análisis de la misma. Después se expone brevemente el papel de las políticas de gobierno dentro de la competencia entre ciudades o regiones, y a manera de conclusión se presentan algunos comentarios finales haciendo una reflexión crítica a la competencia entre territorios y la competitividad.

\section{La competitividad territorial y sus determinantes}

La competitividad territorial, refiriéndonos con ello de manera genérica a los ámbitos regional y urbano, así como las fuentes o factores de la competitividad, adquirió importancia en distintos círculos desde que se concibió a las ciudades y regiones como entes que actuaban y competían por Inversión Extranjera Directa (IED) e inversión doméstica, por acceso a los mercados internacionales, por capital humano, por ciencia y tecnología, e incluso por recursos extra económicos, lo que en turno se reflejaba en el éxito o fracaso económico de dichas ciudades y regiones. Se derivó, a partir de ello, un paradigma alrededor de la competitividad y un debate bastante álgido y rico acerca de su aplicabilidad y conveniencia para los territorios. Paralelamente se fueron desarrollando diversas metodologías de medición y clasificación (Turok, 2004; Begg, 1999; Gordon, 1999; Storper y Manville, 2006). En trabajos tales como Kresl (1995) y Cheshire y Carbonaro (1996) es que se empiezan a definir e identificar los principales factores de competitividad de las ciudades en el ámbito europeo. Por su parte, los índices de competitividad empezaron a destacar como variable de referencia de empresarios, gobiernos y otros agentes económicos para sus decisiones de localización, gestión y acción (Kresl, 1992).

Después de cerca de dos décadas existe ahora vasta literatura sobre el concepto, los determinantes y la forma de medir la competitividad terri- 
torial. Aunque su acepción moderna está asociada con los niveles de productividad o eficiencia relativa de las empresas, la noción de competitividad como origen del comercio inter e intra nacional fue considerada desde los tiempos de Adam Smith. En La riqueza de las naciones, Smith propone como fuente de competitividad las ventajas absolutas, es decir, las naciones podían exportar y competir en aquellos bienes en los que, debido a sus ventajas naturales, eran producidos a un menor costo. Por su parte, David Ricardo destacó la importancia no de las ventajas naturales, sino de las tecnológicas para establecer relaciones de comercio internacional, las cuales estarían basadas en la productividad del trabajo. Heckscher y Ohlin en sus trabajos sobre comercio internacional propusieron también que las ventajas comparativas eran la mejor estrategia de competitividad de los países en el comercio internacional, tomando en cuenta no sólo la productividad de la mano de obra o su bajo costo, como hizo Ricardo, sino también las ventajas derivadas de la abundancia relativa de factores. Por otro lado, Porter (1991) introduce finalmente el concepto de ventajas competitivas. En su trabajo, argumenta que la razón para que las economías nacionales puedan competir en el mercado internacional es a partir de las ventajas provenientes de la innovación y adaptación tecnológica en el proceso productivo, la diferenciación en los productos y los rendimientos crecientes a escala, como resultado de la aglomeración. Señala que aunque las empresas son las que compiten en la escena internacional, la nación funge como un actor que proporciona las condiciones para el desarrollo de este tipo de ventajas (Porter, 1991).

A pesar que el concepto de competitividad se utilizó de forma explícita inicialmente en el ámbito empresarial, la expansión de este concepto alcanzó, como hemos señalado, un estatus como elemento analítico no sólo para las naciones y empresas sino para regiones y ciudades. A este respecto Kresl (1992; 1995) (Kresl y Singh, 1999) y Cheshire y Carbonaro (1996) argumentan que en la esfera internacional la competencia económica no sólo sucede entre las empresas y las naciones, sino que las regiones y ciudades compiten por la atracción y retención de recursos debido a que el entorno local afecta el desempeño de las empresas (JensenButler, 1999).

Generalmente las definiciones de competitividad regional y urbana destacan dos aspectos: la calidad de vida (Camagni, 2002; Storper, 1997; Batey y Frederich, 2000; Porter, 1999; Poot, 2000; Lever y Turok, 1999; Begg, 1999) y la productividad (Camagni, 2002; Porter, 1991; 1999; Krugman, 1994; Begg, 1999; Storper, 1997) como parámetros básicos. Porter (1999) señala que el objetivo principal de una nación es conservar un alto crecimiento y calidad de vida para sus ciudadanos, lo cual depende de la productividad del capital y la mano de obra. Es decir, la alta 
productividad es indicativa de competitividad pero se extiende a un ulterior objetivo, que es el bienestar o calidad de vida. Krugman (1994) argumenta que la competitividad no es más que el incremento de la productividad, así, la mejora en la calidad de vida depende básicamente de la habilidad para incrementar el producto por trabajador.

Por su parte, Lever y Turok (1999: 792) ofrecen una definición de competitividad haciendo referencia al caso específico de las ciudades, y la conceptualizan como: "El grado en que [una región o ciudad] pueden producir bienes y servicios que satisfagan los requerimientos de los mercados internacionales y, simultáneamente, mantener o expandir los ingresos reales, de sus ciudadanos" (President's Commission on Industrial Competitiveness, 1985: 5). Una definición de amplio uso es la de la OCDE (1995), que aunque se refiere a la competitividad de los países, ha sido también empleada para regiones y ciudades. Ahí se define a la competitividad como: "El grado en que, bajo condiciones de libre mercado, un país (región o ciudad) puede producir bienes y servicios de calidad de acuerdo a las exigencias de los mercados internacionales, y mantener, simultáneamente, el crecimiento sostenido de la renta real de los ciudadanos".

Se presenta así una complejización conceptual ya que la competitividad territorial, además de la productividad, abarca cuestiones como el bienestar, la calidad de vida e incluso la preservación del medio ambiente (Cuervo, 2000). En este sentido, se reconoce que el concepto de competitividad a nivel regional y urbano es difícil de definir (Lever, 1999).

Con una visión más holística, Camagni (2002) considera a la región como un ente capaz de ser competitivo en función de su infraestructura tecnológica, social e institucional, que beneficia a las empresas ya que, si bien, son las empresas las que se enfrentan en los mercados globales, éstas son exitosas gracias a la influencia de su entorno regional. Storper (1995; 1997) le confiere especial importancia a las economías de aprendizaje como un medio para alcanzar la competitividad regional. Al igual que Camagni, Storper ve a la competitividad como una relación entre las empresas y las regiones en que ellas se localizan. Específicamente, la define como "La habilidad de una economía para atraer y mantener empresas con un mercado creciente y estable en una actividad; mientras mantiene o incrementa los estándares de vida de aquellos que participan" (Storper, 1995: 286). Así, el espacio se torna en un elemento fundamental ya que, efectivamente, regiones, territorios y ciudades compiten, pues poseen y crean las condiciones que influyen sustancialmente en el desempeño de las actividades económicas (Boshma, 2004; Camagni, 2002; Storper, 1997; Kresl, 1992; Sobrino, 2003; Lever y Turok, 1999, Jensen-Butler, 1999). 
Paralelamente existe una discusión acerca de los factores que explican y contribuyen a la competitividad territorial. Kresl y Singh (1999) retoman los factores determinantes de la competitividad definidos por Kresl (1995), los cuales son clasificados en dos tipos:

1. Económicos: se refiere a aquellos elementos que atañen directamente a los niveles de producción, infraestructura, localización, estructura económica y amenidades urbanas.

2. Estratégicos: son aquellos tales como la efectividad del gobierno, la estrategia urbana, la cooperación público-privada y la flexibilidad institucional.

Porter (1991) destaca el diamante de competitividad según el cual sus determinantes se pueden estructurar a través de cuatro aristas:

1. Condiciones de los factores: capital humano, infraestructura física, administrativa, de información científica y tecnológica y recursos naturales.

2. Condiciones de la demanda: mercado interno y externo.

3. Condiciones de los sectores relacionados y de apoyo: la existencia de proveedores, la presencia de firmas en sectores relacionados y la formación de clusters.

4. Condiciones de estrategia, estructura y rivalidad de la empresa: las condiciones locales que fomentan la inversión e innovación, así como las instituciones que aseguren una competencia abierta entre las firmas.

Begg (1999) desarrolla un esquema en el que el desempeño regional y urbano se ve influenciando por los siguientes factores, que permiten el incremento de los niveles de empleo y productividad:

a) Tendencias sectoriales e influencias macroeconómicas tales como las políticas públicas, la estructura y el desempeño de la economía a nivel nacional en el comportamiento de los sectores económicos regionales y de las ciudades.

b) Características de las compañías en aspectos como créditos, la especialización y los patrones de decisión de los empresarios.

c) Ambiente empresarial que considera aspectos fuera del alcance de los empresarios pero que influyen de manera importante en el desempeño de la empresa; por ejemplo, la disponibilidad de insumos, sus costos y la existencia de mano de obra calificada, además de aspectos sociales como la seguridad y calidad de vida en la ciudad. 
d) Innovación y aprendizaje incluyen aquellos factores que permiten crear un stock de capital intangible en forma de conocimiento, así como la capacidad para fomentar la innovación tecnológica empresarial; también la accesibilidad al soporte de centros de investigación y universidades. Esto puede ser potenciado por la capacidad de mayor atracción de IED, ya que ésta dinamiza la innovación.

Asimismo, Begg resalta la importancia de la participación gubernamental a través de políticas que mejoren cada uno de estos aspectos.

Por último, el esquema de la pirámide de competitividad de Lengyel (2004) distingue cuatro niveles y, al igual que Begg (1999), ubica a la calidad de vida como fin último, mientras que la productividad y las tasas de empleo son vistos como indicadores de competitividad revelada (figura I). Es decir, una mejor calidad de vida es el objetivo máximo alcanzable mediante la mejora en los componentes de la base de la pirámide que son:

a) Categorías básicas. Éstas miden la competitividad revelada e incluyen el nivel de producción, la productividad del trabajo, el empleo y el grado de apertura de la economía. Es importante resaltar que aunque están en el mismo nivel, se hace una diferenciación pues el incremento en el producto es resultado de un incremento en los otros tres factores de esta categoría.

b) Factores de desarrollo. Son factores con un impacto inmediato en las categorías básicas. Incluyen el desarrollo de investigación y tecnología, infraestructura, capital humano, la IED, las Pequeñas y Medianas Empresas (PyMEs), las instituciones y el capital social.

c) Los determinantes del éxito. Son la base de la pirámide y afectan de manera indirecta a las categorías básicas, aunque tienen un efecto de más largo plazo y su significancia va más allá de la política económica. Aquí se incluye la estructura económica, la innovación, la accesibilidad regional, las habilidades de la fuerza de trabajo, los centros de decisión, el ambiente y la identidad regional.

Se observan algunas coincidencias generales en las distintas propuestas, sin embargo, Porter enfatiza la importancia de la formación de clusters, esto es, de la condición de los factores relacionales y de apoyo, así como de las instituciones que aseguren la competencia. En el planteamiento de Lengyel (2004) se hace un esfuerzo por separar los factores y los resultados de la competitividad; aunque no señala, como lo hace Begg (1999), la acción directa del gobierno, la influencia de las tendencias macroeconómicas, ni tampoco de la seguridad, éstas van implícitas en la categoría instituciones y capital social y ambiente. 


\section{Figura I}

\section{Modelo piramidal de competitividad regional}

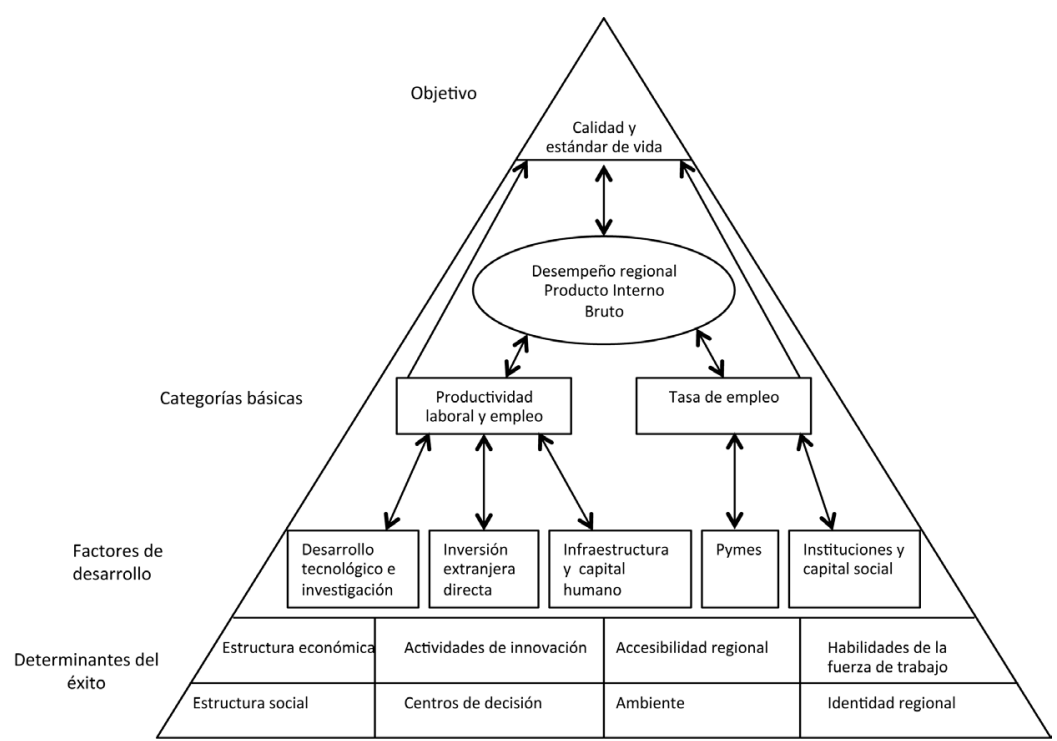

Fuente: Lengyel (2004).

Camagni (2005: 153), al señalar que las ciudades competitivas son aquellas especializadas en, por lo menos, un sector de actividad económica y tienen capacidad de exportación, argumenta que:

la verdadera base de exportación de una ciudad reside en la creatividad de sus universidades y centros de investigación, en la satisfacción de sus empresas de ingeniería y de sus instituciones financieras; en la capacidad de persuasión de sus agencias de relaciones públicas y publicidad, en la flexibilidad de sus redes de transporte y servicios públicos, es decir, en la densa infraestructura de actividades de servicios que permite una fácil movilidad de los factores productivos, su continua y rápida reconversión en producciones siempre nuevas.

Sobrino (2003) presenta algunas otras clasificaciones de los factores de competitividad y, en general, coincide con varios elementos existiendo una convergencia en el seńalamiento de la importancia del capital humano, la infraestructura, las instituciones y la capacidad de aprendizaje que se reflejan en el incremento de la productividad y el empleo.

En resumen, quizá la propuesta más completa es la de Lengyel (2004), pues de una u otra forma engloba los factores considerados por otros autores, pero además, distingue determinantes y resultados, permite visualizar la forma en que están interrelacionados, y permite formular en el marco de la competitividad la existencia de la competencia territorial, la cual se aborda enseguida. 


\section{Teorías y conceptos de competencia regional}

En la literatura acerca de la competitividad territorial no siempre se aborda expresamente la competencia territorial, de ahí que sea este un concepto comparativamente menos investigado desde el punto de vista teórico y empírico, ya que según Batey y Friedrich (2000), el desarrollo de teorías de competencia territorial es difícil. En general, los estudios de competencia entre territorios pueden agruparse en tres:

1. En el primero se encuentran aquellos que consideran la competencia como un mero mecanismo de mercado en el que las actividades económicas se distribuyen a través de las regiones. Esta perspectiva proviene fundamentalmente de autores alemanes, por ejemplo Parr (1978) y Maneval (2000) quienes se basan en las teorías de localización.

2. En la segunda perspectiva se encuentran los que estudian la denominada tax competition refiriéndose a la estrategia de atraer inversión y recursos humanos reduciendo o minimizando el nivel impositivo o creando preferencias impositivas; ha sido desarrollada principalmente por autores de Estados Unidos dado que, por su autonomía, el gasto de los gobiernos locales de ese país en incentivos monetarios y ayudas para la localización de nuevas empresas ha representado cantidades exorbitantes (Thomas, 2003; 2010). Mecanismos similares -formales o informales- han sido utilizados en otros países conduciendo al debate de los efectos reales y los problemas de eficiencia de estos mecanismos de atracción de inversiones.

3. En el tercer enfoque se encuentran aquellos trabajos que consideran a la competencia como un proceso mediante el cual las regiones buscan incrementar su competitividad, enmarcando así todo tipo de acciones que se emprendan, pero principalmente aquellas que fortalezcan las capacidades competitivas locales más allá del favorecimiento fiscal y otros incentivos monetarios. Esta vertiente ha sido desarrollada principalmente por autores de Europa Occidental, probablemente porque la Unión Europea, a diferencia de Estados Unidos y Canadá, tienen fuertes regulaciones para evitar que los gobiernos locales compitan sólo con incentivos monetarios e incurran en la denominada race to the bottom que puede llevar a una competencia ineficiente por la atracción de recursos externos (Thomas, 2010). 
Así, se pueden encontrar distintos conceptos que resaltan diferentes elementos, lo que define la diversa naturaleza de las aplicaciones empíricas. Por ejemplo, los trabajos dentro del primer enfoque elaboran modelos tomando como variable la distribución del Producto Interno Bruto (PIB) entre las regiones de un país; entre estos trabajos se encuentran Márquez y Hewings (2003); Márquez et al, (2007); y Postglione y Hewings (2008). Los trabajos referentes a la segunda perspectiva tratan de encontrar el efecto de los diversos incentivos fiscales en las decisiones de localización de las firmas, el bienestar local, la eficiencia, los salarios y el medio ambiente; se pueden mencionar los trabajos empíricos de De Bartolome y Spiegel (1995), Fisher y Peters (1998), y Thomas (2000); y otros más teóricos como Oates y Schwab (1988), y Ottaviano y Van Ypersele (2005). Los trabajos de Thomas (2000) y (2010) ofrecen numerosa evidencia de incentivos concedidos por parte de gobiernos locales. En el tercer enfoque, entre los trabajos teóricos se encuentran Cheshire y Gordon (1998), Budd e Hirmis (2004), y Chien y Gordon (2008); los trabajos empíricos están más bien orientados a medir la competitividad y hablan de la competencia sólo de manera tangente. Analíticamente, el tercer enfoque es más inclusivo ya que considerar a la competencia sólo como un proceso de mercado o como la sola acción de otorgar incentivos y exenciones fiscales son visiones muy restringidas.

Un concepto que representa de una manera explicita la naturaleza de la competencia territorial es el ofrecido por Cheshire (1999: 843) quien la define como:

un proceso mediante el cual los grupos, actuando en nombre de una economía regional o subregional (normalmente la de una ciudad o región), tratan de promoverla como lugar para la actividad económica ya sea implícita o explícitamente en la competencia con otras áreas. Esta actividad competitiva esta a menudo dirigida a la atracción de inversiones móviles, a veces con la discriminación entre las actividades más y menos deseables.

Es decir, se presenta la competencia como un proceso en el que se promueve a la ciudad o territorio como una mejor localidad para la actividad económica. De forma similar, Gordon (1999) describe la competencia como una mera estrategia de promoción de las regiones y no necesariamente como un proceso de creación de capacidades competitivas. Sin embargo, esta actividad de promoción puede ser equiparada con otras estrategias y acciones de gobierno para la atracción de inversiones, sobre todo en las actividades económicas más deseables, lo que implica la definición de política económica por parte de los gobiernos locales, estatales e incluso el federal o central, a fin de promover el desarrollo de la actividad económica en una localidad específica. 
Según Kresl (1992), las ciudades compiten agresivamente unas contra otras. Compiten por la localización de infraestructura, de actividades gerenciales, de organizaciones internacionales, por conexiones de transporte y por convertirse en punto clave del sistema urbano regional, nacional e internacional. En el planteamiento de Kresl la búsqueda del mejoramiento en la infraestructura y las amenidades urbanas (factores económicos) instan a los gobiernos locales a buscar la atracción de inversión pública del gobierno central (factor estratégico), surgiendo también una competencia directa por recursos fiscales, dado el impacto sobre el crecimiento que esta inversión puede generar en la economía local. Poot (2000) señala que el deseo de crecimiento de las regiones guía a una competencia directa por recursos, personas y mercados. Entre tanto, Budd (1998) afirma que las ciudades compiten para tener mejores factores locacionales, es decir, economías de localización y urbanización, y que la competencia territorial incluye políticas de desarrollo local. Este autor introduce a la discusión el papel del Estado a través de políticas de desarrollo local, coincidiendo en este sentido con Cheshire (1999) y D’ Arcy y Keogh (1998).

Por su parte, Lever (1999) plantea que los principales objetivos de la competencia entre ciudades son la atracción de recursos y de inversión, el crecimiento del producto o valor agregado, la formación de nuevas empresas, población (en el entendido que entre mayor sea la población en una ciudad esto representa mayor capacidad de capital humano, nivel de demanda y poder político), la competencia por fondos públicos y, por último, la competencia por la atracción de eventos internacionales con base en la capacidad de infraestructura de la ciudad. Esto se deriva de la falta de mecanismos regionales de ajuste automático como los que existen para los países -flexibilidad de precios, salarios, devaluación de la moneda-, lo que provoca que los ajustes sucedan a través de la dotación de factores móviles (Camagni, 2005). En consecuencia, entre mayor sea la movilidad de los factores más severo será el nivel de competencia. Esto puede ser fácilmente reflejado en la cantidad de población migrante que reciben las ciudades con mejor desempeño, lo cual puede fungir como un indicador de los resultados de esta competencia.

Camagni (2002) señala que los territorios compiten tanto por la atracción de inversiones extranjeras como en la definición de un papel productivo en el contexto local y global de la división internacional del trabajo. De ahí que entre las estrategias de competencia se considera la especialización, lo que lleva a definir un papel específico en el sistema urbano-regional, nacional o internacional, coincidiendo con Kresl (1992). Este proceso considera no sólo el rol del territorio como proveedor de un ambiente competitivo, sino también el desempeñado en el proceso de 
acumulación del conocimiento y el desarrollo de códigos interpretativos, modos de cooperación y decisiones en los cuales está basado el progreso.

No obstante, la competencia se lleva a cabo no sólo entre regiones del mismo perfil productivo y especialización. También ocurre entre aquellas de diferente especialización productiva cuando las mismas se encuentran cercanas geográficamente y se incrementa en la medida que crece su integración económica interna (Funck, 2000; Cheshire, 1999). La competencia también aumenta con la liberalización comercial y la integración económica con otros países (Kresl, 1992). Ejemplos de ello son la integración de la Unión Europea, la firma del Tratado de Libre Comercio de América del Norte (TLCAN), la Organización Mundial del Comercio (oMC) y otros convenios que facilitan el comercio y la movilidad de capitales a escala global, lo que hace que los gobiernos tengan una mayor necesidad de generar ventajas. Estas capacidades no son requeridas exclusivamente para fungir como líderes en los contextos sub-nacionales o globales sino que se han hecho cada vez más necesarias para la supervivencia ante las presiones impuestas por la globalización.

La competencia territorial sucede como resultado de la búsqueda de una mejora en las condiciones con respecto a otros territorios; es decir, se compite por mejorar estas capacidades de forma más rápida que otras regiones o ciudades, particularmente que aquellas con las que compiten en los mismos mercados en la provisión de bienes y servicios. Esto denota la relatividad intrínseca del proceso de competencia porque ciertamente las administraciones gubernamentales y demás actores locales intentan mejorar la infraestructura, incrementar el desarrollo de ciencia y tecnología, mejorar las amenidades que ofrecen, incrementar la capacitación de la mano de obra, etcétera, en aras de ser ganadores de recursos productivos o ganar en el mercado de los votos, en el caso de los gobiernos. Sin embargo, en el contexto de la competencia territorial, estos esfuerzos no adquieren relevancia si los territorios no son capaces de incrementar sus atributos y capacidades en mayor medida o más rápidamente que otras; es decir, serán ganadores en el proceso de competencia aquellos que desarrollen más rápidamente su capacidad de atracción.

La competencia puede tener resultados de suma cero (Cheshire y Gordon, 1998; Camagni, 2005), de fomento del crecimiento o suma positiva (Cheshire y Gordon, 1998) o de suma negativa (Rodríguez-Pose y Arbix, 2000) dependiendo en buena medida del tipo de acciones que se lleven a cabo. Potter (2009) afirma que cuando una región crece puede impactar en forma positiva a sus vecinos regionales o potenciar el crecimiento dentro de su sistema territorial. Este efecto podría incrementarse en función de la capacidad de cooperación y concertación de actores económicos y políticos al permitir la consecución de economías de com- 
plementariedad o sinergia en los sistemas urbanos (Camagni, 2005). Un ejemplo es la especialización de una economía que puede generar especializaciones complementarias en territorios aledaños. Esto, en cierta forma, justifica el apoyo a ciudades más débilmente competitivas ya que, según Jensen-Butler (1999) y Camagni (2002; 2005), no hay una pelea entre equidad y eficiencia en el mediano y largo plazo debido a estos efectos positivos entre regiones.

La competencia tiene además efectos de largo plazo ya que, en general, aquellos territorios que históricamente han tenido mejor desempeño, tienden a crecer más rápido debido a su mayor población y stock de capital generando procesos acumulativos (Cheshire y Carbonaro, 1996). Es decir, además de las estrategias de los gobiernos, el desempeño de los actores privados y de las instituciones, influye el trasfondo histórico y las dotaciones iniciales, por ello se vuelve fundamental el entorno socioeconómico y su evolución en el tiempo.

En suma, según los autores revisados, la competencia territorial puede definirse como un proceso en el cual las regiones compiten primordialmente por la atracción y retención de recursos diversos ya que con ello es factible alcanzar objetivos de competitividad revelada, así como objetivos políticos y sociales de desarrollo (Batey y Frederich, 2000; Poot, 2000). Se trata de un proceso en el que los actores, en nombre del territorio, emprenden acciones para incentivar su atractivo y calidad, y donde la formulación de políticas locales puede ser fundamental (Cheshire, 1999; Budd, 1998).

Considerando el modelo piramidal de Lengyel (2004), es en los Factores de desarrollo y en los Determinantes del éxito donde los territorios compiten, ya que tratan de mejorar en uno o más aspectos de éstos, los que posteriormente impactan la productividad, el empleo y la producción, medidas éstas de competitividad revelada y, que al final, podrían mejorar la calidad de vida. Sin embargo, generalmente en la práctica, las expectativas de la competencia regional quedan limitadas a la retención y la atracción de factores productivos como la IED, la inversión pública, la inversión privada nacional y los recursos humanos; también al soporte electoral de la sociedad y a la popularidad de los gobiernos locales sin que necesariamente se persiga la mejora en el bienestar de la población.

Se observa que aunque se han vislumbrado aspectos relevantes tales como los participantes, las estrategias y acciones, y los objetivos de la competencia, su definición no ha sido consistente ni claramente formulada. Persiste la necesidad de un concepto más integral y menos ambiguo, mismo que, a la luz de los diferentes estudios revisados, es posible lograr en aras de la delimitación de un marco analítico para el estudio de la competencia territorial. 
Aquí definimos la competencia territorial como el proceso mediante el cual los territorios, a través de distintos actores públicos y privados, establecen una variedad de estrategias y acciones intentando promoverse en los mercados locales y externos. Asimismo, atraer recursos -fondos públicos, inversión privada, recursos humanos, tecnología- en mayor medida que otros territorios, principalmente más que aquellos con los que compiten directamente en la producción de bienes y servicios, a fin de lograr una mejora en los indicadores de competitividad revelada o incluso de desarrollo y bienestar. De esta manera, mientras que la competitividad es mayoritariamente definida como una capacidad (oCDE, 1995), habilidad (Storper, 1995) o grado-medida (Lever y Turok, 1999) de competir y generar bienestar, la competencia es el proceso detrás, en donde las acciones y relaciones emprendidas hacia la mejora competitiva son cruciales.

A fin de lograr la construcción de una estructura lógica que considere los aspectos más ilustrativos de la competencia regional, es preciso considerar que dentro de la competencia territorial puede participar una diversidad y multiplicidad de actores (gobiernos, instituciones y actores privados). De dichos actores y de sus relaciones se derivan los tipos de competencia y sus resultados. Asimismo, es útil distinguir sus objetivos ya que evidentemente pueden ser varios y diversos. Entre estos se encuentran el dominio de mercados-locales, nacionales o globales- (Poot, 2000); la atracción de recursos móviles - capital y trabajo- (Camagni, 2002; Lever, 1999; Poot, 2000; Batey y Friedrich, 2000); la obtención de mayores dotaciones de infraestructura pública (Budd, 1998; Kresl, 1992); ocupar un lugar específico en la cadena de producción regional, nacional o internacional (Kresl, 1992; Camagni, 2002); incrementar los niveles de producción (Lever, 1999); aumentar la participación relativa en el producto nacional (Maneval, 2000); la captación de fondos públicos (Lever, 1999); y la captación de votos ciudadanos (Johansson, 2000).

A partir de la revisión de los distintos planteamientos previos encontrados en la literatura, enseguida se propone una estructura analítica que sirva para sistematizar el estudio de procesos de competencia regional o urbana.

\section{Caracterización de la competencia regional}

En el análisis de la competencia territorial es útil partir de un esquema básico que sirva para abordar los aspectos relevantes y sistematizarlos. En la definición de tal esquema es de vital importancia incorporar todos aquellos elementos contextuales propios a cada caso. A pesar de que la literatura dominante en el tema proviene del mundo desarrollado debido 
a lo extendido del paradigma de la competitividad derivado a su vez del de la globalización, la competencia territorial ha permeado en distintas latitudes en el orbe, por lo que se presentan diferentes tipos de procesos dependiendo de las características de los sistemas sociales, económicos y políticos particulares. Batey y Friedrich (2000) proponen una mezcla de elementos referentes a efectos de externalidades, mecanismos no monetarios de mercado, y no sólo a los mecanismos estrictamente pecuniarios. La estructura por ellos sugerida parte de cuatro elementos eje o características básicas del proceso de competencia: a) el tipo de competencia, b) el tipo de competidores, c) el tipo de relaciones entre competidores, y d) los resultados. Los tres primeros ejes componen lo que podría denominarse estructura del proceso de competencia que lleva luego a un resultado, es decir, el punto d). Esto se resume en la figura II.

\section{Figura II}

\section{Marco analítico de la competencia territorial}

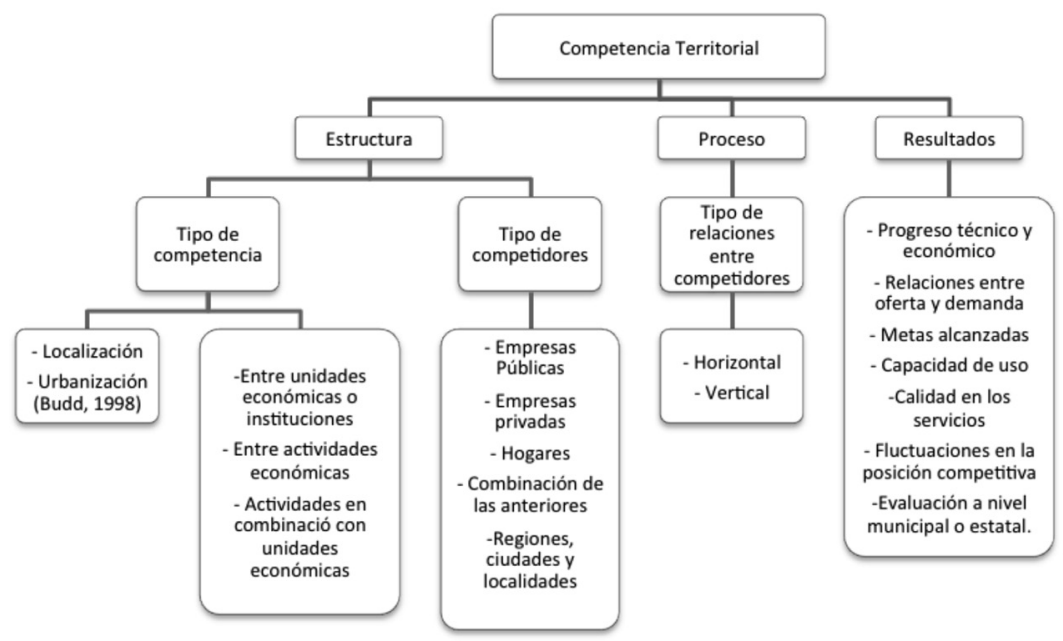

Fuente: Elaboración propia con base en Batey y Friedrich (2000), Budd (1998).

A continuación se detalla en qué consiste cada uno de estos elementos:

\subsection{Tipos de competencia}

Batey y Friedrich (2000) proponen los siguientes tipos de competencia:

1. Competencia entre agentes económicos o instituciones en las regiones respectivas. 
2. Competencia entre actividades económicas en procesos de asignación de recursos y factores de la producción.

3. Actividades en combinación con unidades económicas.

A esta sugerencia sumamos la de Budd (1998) para quien existen dos tipos de competencia territorial:

1. Competencia entre actividades económicas o mercados, que operan desde ciudades región, es decir, economías de localización o externalidades positivas en un sector o mercado específico.

2. Competencia en términos de las características de las ciudades o regiones y su capital social, esto es, las economías de urbanización, por ejemplo, la infraestructura, la calidad y disponibilidad de educación.

La propuesta de Batey y Friedrich (2000) es más completa debido a que contempla la participación de actores públicos y privados que pueden actuar individualmente o bien como parte del tejido productivo y social. Esto significa que la competencia depende tanto de las acciones de gobierno, entendidas como políticas de inversión públicas y estrategias competitivas formales y no formales -en ocasiones con el acuerdo e iniciativa de la sociedad- así como de estrategias de las empresas en cada ciudad, las cuales son guiadas también por el entorno productivo para su localización.

\subsection{Tipos de competidores}

El tipo de competidores se refiere a las entidades participantes en el proceso de competencia territorial. De ellos dependen los objetivos perseguidos y la forma en que los resultados son medidos. En general, se trata de empresas privadas, agencias públicas, hogares o una combinación de los mismos

Las empresas compiten directamente por mercados finales o intermedios; las oficinas públicas por la atracción y retención de inversiones. También pueden competir las oficinas públicas entre sí dentro de la misma ciudad por una mayor asignación de recursos (que en términos políticos se traduciría en mayor éxito electoral). Por su parte, los hogares privados compiten en el mercado inmobiliario por servicios, infraestructura, amenidades, etc. También pueden ser considerados como competidores los estados en una federación, provincias, municipalidades, o países dentro de bloques regionales, o bien otras unidades económicas localizados en ellos, como las empresas. 


\section{Figura III}

\section{Estructura de la competencia en función de los competidores,} campos de acción y objetivos

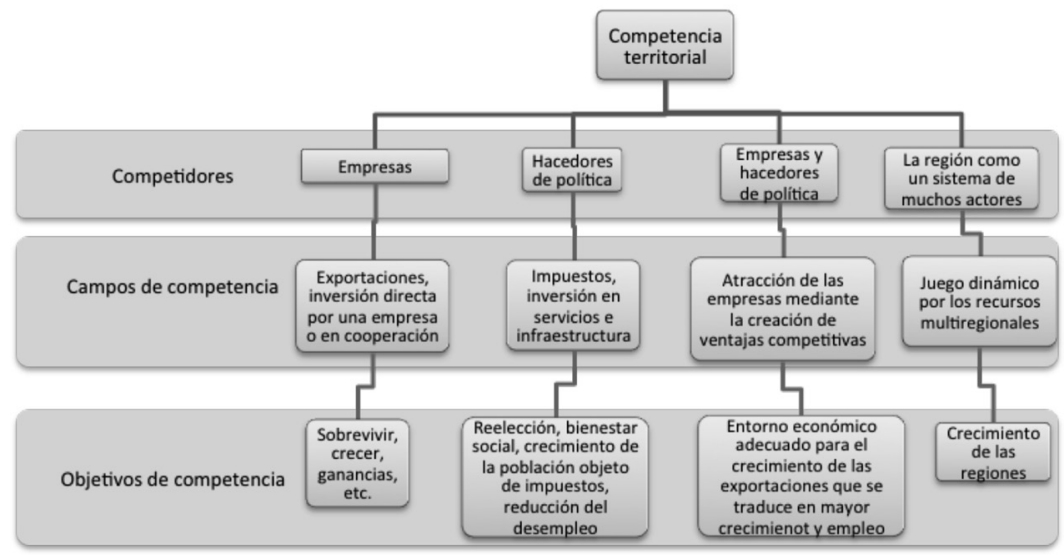

Fuente: Johansson (2000: 37).

Johansson (2000) presenta, de forma sintética, otro esquema (figura III) de los tipos de competidores, el medio de competencia y los objetivos de la misma, cuya naturaleza es similar al planteado por Batey y Friedrich.

Este último planteamiento coincide con lo dicho por Kresl (1992), Lever (1999), Camagni (2005), Cheshire y Gordon (1998) y Potter (2009) al considerar el territorio como un sistema en el cual participan diversos actores y el medio de competencia, un juego relativo y dinámico por los recursos multiregionales.

\subsection{Tipos de relaciones entre los competidores}

Batey y Friedrich (2000) identifican dos tipos de relaciones de competencia: horizontal y vertical. La competencia horizontal se lleva a cabo entre actores que se encuentran en el mismo nivel, es decir, empresas contra empresas, gobiernos locales contra gobiernos locales, etcétera. Mientras que la competencia vertical se suscita entre actores en distintos niveles jerárquicos y de autoridad como empresas contra personas, empresas contra gobiernos, o bien, gobiernos en diferentes niveles como el gobierno local contra el gobierno estatal o federal.

El tipo de parámetros de acción elegidos por los competidores determina la relación de competencia y, en función de esto, surgen diferentes campos de lucha, por ejemplo: 
a) Competencia asociada con el dominio de las compras y ventas en los mercados regionales, nacionales e internacionales.

b) Competencia por la localización para el uso de recursos.

c) Competencia en la gestión de externalidades.

d) Competencia en coordinaciones intergubernamentales en algunas tareas y producción de bienes.

e) Competencia en la elaboración de políticas, mandatos, posiciones, etcétera, provocando conflictos de tipo político.

f) Competencia política para gobernar una región o para tomar el papel como responsable dentro de la competencia regional. (Batey y Friedrich, 2000).

De esta manera, emergen tipos de competencia específicos, mismos que definen las relaciones entre los actores. En el caso de los gobiernos, sus parámetros de acción pueden ser de tipo macroeconómico, esto es, a través del gasto, ingreso por impuestos, deuda, subvenciones y empleos. Asimismo, pueden utilizar algunos parámetros de acción microeconómica como: tasas de impuestos y tarifas, proyectos individuales, o proyectos de localidades y regiones. En el caso de empresas privadas o públicas, sus parámetros de acción son de tipo microeconómico como precios, cantidad de producción, servicio, calidad, ubicación y crédito. Por su parte, los hogares tienen parámetros mayoritariamente microeconómicos.

\subsection{Resultados de competencia}

Los resultados de la competencia pueden ser evaluados en función de los objetivos de los competidores.

Entre los posibles indicadores a considerar se encuentran:

a) Progreso técnico y económico. Cambios en infraestructura, formación de conocimiento y capital humano, establecimiento de empresas de alta tecnología, atracción de investigadores, formación de clusters y polos de desarrollo.

b) Relaciones entre la oferta y la demanda. Productividad, relación de producción de base exportación respecto a la producción que no es de base exportación.

c) Indicadores económicos. Ingreso, producción, ingresos por impuestos, número de empresas atraídas y distribución del ingreso.

d) Costos. Disminución de costos de la mano de obra, de las comunicaciones y de tasas impositivas.

e) Capacidad de uso. Empleo regional, capacidad de los parques industriales. 
f) Calidad de los servicios. Valor residencial, costo del ocio y tiempo libre, calidad del ambiente, flexibilidad de las instituciones políticas.

g) Fluctuaciones en la competencia. Migración, número de competidores, cambio en la jerarquía, reformas funcionales y territoriales, cambio en las posiciones competitivas.

h) Evaluación a nivel municipal o estatal. Función de bienestar de la región, objetivos económicos y políticos, posición en la competencia dentro de la región, reducción de la migración, base exportación.

i) Evaluación en el sentido de las unidades económicas individuales. Ganancias, ingreso, infraestructura y carga fiscal.

Otras medidas de resultados que se han propuesto en la literatura son el cambio en la participación en la producción regional (Maneval, 2000) y el nivel de eficiencia de la economía (Reggiani et al., 2000). Sobrino (2003) propone un índice de competencia que básicamente mide el crecimiento de las regiones ponderando aspectos varios como el tamaño de la economía, su crecimiento absoluto y su crecimiento relativo.

La combinación de diferentes parámetros asociados con los distintos componentes de la estructura de competencia territorial da pauta a variadas formas y resultados que pueden presentarse en contextos sociales, políticos y económicos diversos. Por otra parte, en este marco general de análisis, la visión de los territorios como un sistema de actores implica el papel trascendente de las estrategias y políticas de gobierno ya que este se constituye en un ente determinante con poder para influir y dirigir las estrategias y los resultados de la competencia territorial. Se presenta a continuación un apartado sobre las políticas de competencia regional y el papel de los gobiernos.

\section{Políticas de competencia territorial}

Generalmente los gobiernos y la política regional, nacional y local se consideran agentes fundamentales en la competencia territorial. La gran mayoría de análisis empíricos intentan evaluar los resultados de la acción gubernamental en distintos niveles, y de la formulación, implementación y resultados de políticas de competencia territorial. En la medida que los gobiernos experimentan las presiones económicas a las se enfrentan los territorios que dirigen, intervendrán para hacer frente a éstas contrarrestando o incentivando la competencia.

Según Potter (2009), la instrumentación de la competencia territorial desde la perspectiva de los gobiernos se lleva a cabo mediante: 
a) Subsidios a las empresas.

b) Planeación nacional, regional o local.

c) El manejo de las externalidades que se presentan en la economía local.

d) Transporte interregional e intra-regional.

e) Apoyo para la formación de clusters.

f) Políticas para la transferencia de conocimiento.

Este autor hace una diferenciación entre las políticas directas e indirectas de competencia y considera como políticas directas aquellas relacionadas con el incremento del capital humano, la innovación y la empresarialidad. Entre las políticas indirectas se encuentran casi todas aquellas tendientes a atraer inversión mediante incentivos.

Por su parte, Kresl (1992) menciona que para ser exitosas las ciudades deben formular una estrategia enfocada a la realidad de su economía. Por lo tanto, deben tener muy claro qué quieren lograr y estar dispuestas a cooperar con otras ciudades para conseguir objetivos específicos que puedan tener en común. En este sentido, coincide con el planteamiento de Camagni (2005) acerca de la formulación de un plan estratégico a fin de retener el capital humano existente y mejorar las capacidades competitivas de la región. Camagni propone una nueva forma de gobernanza en las economías locales, de manera que puedan seguirse planes que lleven a la ciudad a una competitividad sostenible en el largo plazo en función de la actuación de diversos actores articulados por los hacedores de políticas.

Kresl (1992) coincide con Camagni (2002), Lever (1999), Begg (1999) y Johansson (2000) en el sentido de que las ciudades deben buscar una especialización que les permita insertarse en la división internacional de trabajo. Así serán parte importante de una red productiva, por lo menos en un bien o servicio, siendo esta la estrategia más importante de competencia ya que, como señala Lever, una ciudad no puede ser competitiva en varias actividades económicas a menos que se trate de una ciudad mundial que compita en diferentes ámbitos. Por tal motivo, Kresl (1992) insta a las ciudades a encontrar nichos de mercado. También plantea que las ciudades deben conseguir ser valoradas como indispensables para los consumidores y productores. De esta manera, los actores privados (empresas y personas) serán menos volátiles. La competencia en el mundo global cada vez tiene menos que ver con los factores tradicionales de localización y ventajas de comercio (factores económicos), y más con recursos humanos y culturales e institucionales (factores estratégicos). Por último, coincidiendo con Camagni (2002; 2005) y Castells (1999), señala que las regiones y ciudades pueden competir con información y conocimiento, ya sea 
generándolos o aplicándolos para la innovación en la producción a través de políticas de competencia enfocadas a estos aspectos.

En cuanto a las derivaciones de las políticas de competencia territorial Cheshire y Gordon (1998) consideran que pueden resultar ser de suma cero, aunque resaltan que puede haber políticas que incentiven un crecimiento conjunto, coincidiendo en ello con Camagni (2002). Para enfrentar la competencia todas las ciudades deben definir una estrategia e incentivar a los agentes locales a que sean más competitivos, siendo este último tipo de políticas, las que generan crecimientos conjuntos en los sistemas urbanos. Como políticas de suma positiva mencionan el entrenamiento empresarial, el fomento de la empresarialidad, la ayuda técnica a las nuevas empresas, la asesoría empresarial, la reducción de la incertidumbre y la infraestructura especializada. Como políticas de suma cero, estos autores, identifican todas aquellas acciones de simple promoción, la atracción de inversiones móviles, los subsidios a la inversión y los subsidios a la localización. En general, Cheshire y Gordon (1998) encuentran que las mejores políticas de competencia son aquellas que se enfocan en incrementar las capacidades de los sectores productivos locales, sobre todo, aquellas que proporcionen flexibilidad en los requerimientos de localización de mano de obra de las empresas.

Rodríguez-Pose y Arbix (2000) señalan las políticas de suma negativa, cuando, con la finalidad de atraer inversiones, las haciendas locales compiten a través de incentivos económicos, provisión de infraestructura, donación de terrenos, etcétera, siendo el único beneficio para la localidad el empleo generado, mientras que el gasto de los gobiernos para la atracción de nuevas inversiones es tal que los lleve a la bancarrota, lo cual afecta no sólo a la localidad sino a todo el país, generando así efectos negativos netos. Este tema no es trivial en ningún sentido, dado que según Fisher y Peters (1998) los gobiernos continúan otorgando esta clase de incentivos, un tanto irracionales, debido a que siguen una inercia de llevar a cabo este tipo de acciones, lo que representa un potencial desperdicio de recursos. Aunado a ello, este tipo de competencia genera polarización dado que las administraciones más ricas son también las que pueden ofrecer los mejores incentivos, de forma tal que la competencia puede ser fuente de desigualdades si no es planeada o regulada estratégicamente desde un nivel central; es decir, no sólo la política territorial desde abajo es la que importa, sino además el papel fundamental de la política espacial desde el nivel central para atender cuestiones de eficiencia y equidad nacionales. Puede haber sesgos en las elecciones racionales y supuestamente estratégicas de los gobiernos nacionales e incluso de las empresas para orientar selectivamente la generación de ventajas y las inversiones (Vainer, 2000). 
Para que las estrategias de competencia no sean de suma cero o negativa es vital el fomento de las relaciones entre las empresas y los centros de investigación y universidades, sin lo cual las empresas, especialmente las extranjeras, no tienen mayor impacto en la economía local (Camagni, 2005). Coraggio (2000), por su parte, destaca la importancia de competir por la gente y no por el capital, y de mejorar las condiciones locales en todos los sentidos a fin de atraer de forma automática nuevas y mejores inversiones, dado que la pura atracción de capital frecuentemente socava el bienestar general. Begg (1999) ańade la importancia de la cooperación entre ciudades en aspectos como problemas ambientales y en la socialización de experiencias de política exitosas así como la capacidad de adaptabilidad y flexibilidad de los territorios ya que esto fomenta la innovación, principal fuente de competitividad. Por adaptabilidad y flexibilidad se refiere a la capacidad para responder a nuevas demandas de infraestructura física, lo cual está en función del nivel de gasto público disponible y la flexibilidad de las instituciones ante las nuevas demandas de los actores privados en la economía. Se trata de una planeación estratégica que puede generar procesos de cooperación más que de competencia entre territorios subnacionales.

En suma, los gobiernos pueden establecer diversas políticas de competencia encaminadas a mejorar la posición relativa de sus territorios. Los resultados dependerán del tipo de políticas emprendidas y de la eficiencia en su implementación. Al respecto han surgido críticas en relación con resultados poco alentadores derivados de la integración de las economías mundiales y las crecientes presiones externas hacia una mayor competencia, como en el caso de América Latina donde la evidencia ha demostrado como resultado reducidas regiones ganadoras y las mayoritarias y empobrecidas regiones perdedoras. Se trata además de una crítica a la visión de los territorios como mercancías o como empresas, y el uso de esta premisa como instrumento ideológico que guía la política territorial (Vainer, 2000).

Por ello, a pesar de que la lógica de la competitividad fue adquiriendo predominio en el discurso de la política pública, existe una historia de iniciativas que han propuesto paradigmas alternativos orientados a alcanzar objetivos de desarrollo territorial (Bristow, 2011; Cochrane, 2011). Esto es debido al fracaso crónico de las políticas nacionales de desarrollo territorial, lo que ha llevado a esfuerzos por contener la corriente dominante en busca del desarrollo local y regional. Algunas de estas propuestas enaltecen la potencialidad de los factores cualitativos, tales como los cognitivos, simbólicos, culturales, cívicos, etc. donde se activan, la solidaridad, la cooperación territorial y la descentralización político-territorial, e incluso la planeación estratégica bajo otra orientación. Boisier (2001: 
7), por ejemplo, señala que el territorio "contiene, en términos reales o en términos potenciales, los factores de su propio desarrollo".

\section{Conclusiones}

La competencia territorial es un concepto que abarca áreas y mecanismos de disputa económica, social e incluso política, bajo el cual se pueden analizar distintos procesos a nivel territorial. Las posibilidades de competencia se han intensificado debido a la naturaleza de las relaciones entre las regiones, determinadas principalmente por una mayor integración económica, que ha flexibilizado la movilidad de factores entre regiones, ciudades y países, pero especialmente del capital y la tecnología. Aquí la competencia territorial ha sido identificada como la expresión de la competitividad traducida en un proceso en que, al buscar incrementar sus capacidades productivas y de dominio en la escena nacional o internacional, se genera una dinámica de acción por parte de agentes económicos, sociales y políticos en los territorios que entran en competencia directa por recursos de inversión pública y privada, fondos públicos y de capital humano, y donde el objetivo se refiere a indicadores de competitividad revelada, pudiendo alcanzar también el de mejorar la calidad de vida y el bienestar social. En la competencia territorial existen competidores, motivaciones, objetivos, acciones, estrategias y resultados en los que el fin último es maximizar los beneficios económicos, políticos y sociales pero donde no siempre se obtienen resultados satisfactorios, especialmente en materia social.

Ha sido objetivo primordial de este documento establecer un marco de análisis y caracterización de la competencia territorial. En él se establecen los distintos componentes de la estructura de los procesos de competencia, una diversidad de parámetros de representación de dichos componentes, así como una multiplicidad de variables de resultados. La combinación entre sí de los diferentes componentes abre la posibilidad de distintos tipos de competencia territorial existentes en marcos sociales, políticos y económicos variados. Dicho marco de análisis permite profundizar en casos específicos e identificar áreas de conflictos, así como la evaluación de resultados. El concepto de competencia regional y su caracterización permiten la sistematización de su estudio y su aplicación a casos de análisis empírico, por lo que se insta a la utilización del mismo para el estudio de los territorios.

Como reflexión última cabe insistir que la competencia y la competitividad son una alternativa, quizá no la mejor en numerosos casos de desarrollo de los territorios y de las sociedades que ellos albergan. En realidad, tanto las regiones como las ciudades enfrentan un campo de 
acción altamente restringido y un panorama agravado debido al decaimiento de la economía global. Seguirá habiendo propulsores, así como escépticos del potencial de la acción local y regional si ésta no se circunscribe a una agenda o programa de cambio dictado desde el nivel global, como lo ha sido la agenda de la competitividad. Es indudable que aún predomina este paradigma a pesar de las limitaciones que plantea. Por lo que resulta fundamental seguir evaluando esta perspectiva en términos de sus alcances y restricciones para explicar el estado de desarrollo de lugares y personas en distintos contextos y las diferencias entre ellos.

\section{Bibliografía}

Batey, Peter y Peter Friedrich (2000), "Aspects of regional competition", en Peter Batey y Peter Friedrich (eds.), Regional Competition, Springer-Verlag, Berlin, pp. 3-33.

Begg, Ian (1999), "Cities and competitiveness", Urban Studies, 36 (5-6), University of Glasgow, Glasgow, pp. 795-809.

Boisier, Sergio (2001), "Desarrollo (local): ¿đe qué estamos hablando?”, en Oscar Madoery y Antonio Vázquez (eds.), Transformaciones globales, instituciones y politicas de desarrollo local, Homo Sapiens, Rosario, pp. 79-109.

Boshma, Ron (2004), "Competitive regions from an evolutionary perspective”, en R. Martin, M. Kitson, y P. Tyler (comps.), Regional competitiveness, Routledge, London and New York, pp. 11-24.

Bristow, Gillian (2011), “Territorial competitiveness and local and regional economic development: A classic tale of 'Theory led by policy”, en Andy Pike, Andrés Rodríguez-Pose y John Tomaney (eds.), Handbook of local and regional development, Routledge, London, pp. 344-355.

Budd, Leslie (1998), "Territorial competition and globalization: Scylla and Charybdis of european cities", Urban Studies, 35 (4), University of Glasgow, Glasgow, pp. 663-685.

Budd, Leslie y Amer Hirmis (2004), "Conceptual framework for regional competitiveness”, Regional Studies, 38 (9), Regional Studies Association, Seaford, pp. 1015-1028. 
Camagni, Roberto (2002), "On the concept of territorial competitiveness: sound or misleding?”, Urban Studies, 39 (13), University of Glasgow, Glasgow, pp. 2395-2411.

Camagni, Roberto (2005), Economia urbana, Antoni Bosh, editor, Barcelona.

Castells, Manuel (1999), La era de la información, Siglo XXI, México.

Cheshire, Paul y Gianni Carbonaro (1996), "Urban economic growth in Europe: testing theory and policy prescriptions", Urban Studies, 33 (7), University of Glasgow, Glasgow, pp. 1111-1128.

Cheshire, Paul y Ian Gordon (1998), "Territorial competition: some lessons for policy", The Annals of Regional Science, 32, SpringerVerlag Berlin Heidelberg, Berlin, pp. 321-346.

Cheshire, Paul (1999), "Cities in competition: articulating the gains from integration”, Urban Studies, 36 (5-6), University of Glasgow, Glasgow, pp. 843-864.

Chien, Shiuh-Shen y Ian Gordon (2008), "Territorial competition in China and the West", Regional Studies, 42 (1), Regional Studies Association, Seaford, pp. 31-49.

Cochrane, Allan (2011), "Alternative approaches to local and regional development", en Andy Pike, Andrés Rodríguez-Pose y John Tomaney (eds.), Handbook of local and regional development, Routledge, London, pp. 97-105.

Coraggio, José (2000), “¿Competir por el capital o competir por la gente? Sentidos alternativos de la política metropolitana”, en Antonio Vázquez y Oscar Madoery (comps.), Transformaciones globales, instituciones y politicas de desarrollo local, Homo Sapiens Ediciones, Rosario, pp. 173-199.

Cuervo, Luis (2000), "Ciudad y competitividad: entre las partes y el todo", Territorios, núm. 3, Universidad de los Andes, Bogotá, pp. 69-80.

D’Arcy, Éamonn and Geoffrey Keogh (1998), “Territorial competition and property market process: an exploratory analysis", Urban Studies, 35, University of Glasgow, Glasgow, pp. 1215-1230. 
De Bartolome, Charles y Mark Spiegel (1995), "Regional competition for domestic and foreign investment: evidence from state development expenditures", Journal of Urban Economics, 37, Elsevier, pp. 239-259.

Fisher, Peter y Alan Peters (1998), Industrial incentives. Competition among american states and cities, Upjohn Institute for Employment Research, Michigan.

Funck, Rolf (2000), "Hard and soft determinants of interrregional competition", en Peter Batey y Peter Friedrich (eds.), Regional competition, Springer-Verlag, Berlin, pp. 66-86.

Gordon, Ian (1999), "Internationalization and urban competition", Urban Studies, 36 (5-6), University of Glasgow, Glasgow, pp. 1001-1016.

Jensen-Butler, Chris (1999), "Cities in competition: equity issues", Urban Studies, 36 (5-6), University of Glasgow, Glasgow, pp. 865-891.

Johansson, Börje (2000), "Regional competition: endogenous policysupported process", en Peter Batey y Peter Friedrich (eds.), Regional competition, Springer-Verlag, Berlin, pp. 34-65.

Kresl, Peter (1992), The urban economy and regional trade liberalization, Praeger Publishers, New York.

Kresl, Peter (1995), "The determinants of urban competitiveness", en Peter Kresl y Gary Gapper (eds.), North american cities and the global economy: challenges and opportunities, Sage Publications, California, pp. 45-68.

Kresl, Peter y Balwant Singh (1999), "Competitiveness and the urban economy: twenty-four large US metropolitan areas", Urban Studies, 36 (5-6), University of Glasgow, Glasgow, pp. 1017-1027.

Krugman, Paul (1994), “Competitiveness. A dangerous obsession”, Foreign affairs, 73 (2), Council of Foreign Relations, New York, pp. 28-44.

Lengyel, Imre (2004), "El modelo piramidal: mejora de la competitividad en Hungría”, Acta Económica, 54 (3), Facultad de Economía, Universidad de Banja Luka, Banja Luka, pp. 323-342. 
Lever, William (1999), "Competitive cities in Europe", Urban Studies, 26 (5-6), University of Glasgow, Glasgow, pp. 1029-1044.

Lever, William y Ivan Turok (1999), "Competitive cities: introduction to the review", Urban Studies, 36 (5-6), University of Glasgow, Glasgow, pp. 791-793.

Maneval, Helmut (2000), "Disarmament in Germany and regional competition”, en Peter Batey y Peter Friedrich (eds.), Regional Competition, Spinger-Verlag, Berlin, pp. 231-246.

Márquez, Miguel y Geoffrey Hewings (2003), "Geographical competition between regional economies: The case of Spain", The Annals of Regional Science, 37, Springer-Verlag, Berlin, pp. 559-580.

Márquez, Miguel, Geoffrey Hewings y Julián Ramajo (2007), “Efectos macroeconómicos dinámicos dentro del sistema regional peninsular español", Libro de actas, VIII Congreso de Estudios Extremeños, Diputación Provincial de Badajoz-Universidad de Extremadura, pp. 1810-1838.

Oates, Wallace y Schwab, Robert (1988), "Economic competition among jurisdictions: efficiency enhancing or distortion inducing?", Journal of Public Economics, 35, Elsevier Publications, North Holland, pp. 333-354.

OCDE (Organisation for Economic Co-operation and Development) (1995), Begg, Iain (1999), "Cities and Competitiveness", Urban Studies, 36, University of Glasgow, Glasgow, pp. 795-809.

Ottaviano, Gianmarco y Tanguy Van Ypersele (2005), "Market size and tax competition", Journal of International Economics, 67, ElsevierUniversity of Wisconsin, Madison, pp. 25-46.

Parr, John (1978), "Regional competition, growth pole policy and public intervention", en Walter Buhr y Peter Friedrich (eds.), Competition among small regions, Nomos Verlagsgesellschaft, Baden-Baden, Germany, pp.122-135.

Poot, Jacques (2000), "Reflection on local economy-wide effects of territorial competition", en Peter Batey y Peter Friedrich (eds.), Regional competition, Springer-Verlag, Berlin, pp. 205-230. 
Porter, Michael (1991), La ventaja competitiva de las naciones, Vergara, Buenos Aires.

Porter, Michael (1999), Ser competitivo, Ediciones Deusto, Bilbao.

Postglione, Paolo y Geoffrey Hewings (2008), "Hierarchical spatial interaction among the italian regions: a nonlinear relative dynamics approach", Journal of Geographical Systems, Springer-Verlag, Berlin, pp. 369-382.

Potter, Jonathan (2009), "Evaluating regional competitiveness policies: insights from the new economic geography", Urban Studies, 43 (9), University of Glasgow, Glasgow, pp. 1225-1236.

President's Commission on Industrial Competitiveness (1985), "Review of findings of the president's comision on industrial competitiveness", Hearing before the Commite on Finance United States, 29 de marzo, Washington.

Reggiani, Aura, Peter Nijkamp y Enrico Sabella (2000), "Evolutionary algorithms for modelling interregional transport flows", en Peter Batey y Peter Friedrich (eds.), Regional competition, SpringerVerlag, Berlin, pp. 159-185.

Rodríguez-Pose, Andrés y Glauco Arbix (2000), "Strategies of waste: bidding wars in the brazilian automobile sector", International Journal of Urban and Regional Research, 25 (1), Blackwell Publishing, Oxford, pp. 134-154.

Sobrino, Jaime (2003), Competitividad de las ciudades en México, El Colegio de México, México.

Storper, Michael (1995), "Competitiveness policy options: the technology-regions connection", Growth and Change, 26 (2), Centro de negocios e investigación económica de la Universidad de Kentucky, Lexington, pp. 285-308.

Storper, Michael (1997), The regional world, The Guilford Press, New York.

Storper, Michael y Michael Manville (2006), "Behavior, preferences and cities: urban theory", Urban Studies, 43 (8), University of Glasgow, Glasgow, pp. 1247-1274. 
Thomas, Keneth (2000), Competing for capital: Europe and North America in a global eva, Georgetown University Press, Washington DC.

Thomas, Keneth (2003), "Geographic scales and the competition for economic growth", American Behavioral Scientist, 46, Sage Publications, Arizona, pp. 987-1001.

Thomas, Keneth (2010), Investments incentives and the global competition for capital, Palgrave Mcmillan, Hampshire.

Turok, Ivan (2004), "Cities, regions and competitiveness", Regional Studies, 38 (9), Regional Studies Association, Seaford, pp. 1069-1083.

Vainer, Carlos (2000), "Pátria, empresa e mercadoria: notas sobre a estratégia discursiva do Planejamento Estratégico Urbano", en Otília Arantes, Carlos Vainer y Ermínia Maricato, A cidade do pensamento único: desmanchando consensos, Editora Vozes, Petrópolis, pp. 75-103.

Recibido: 8 de mayo de 2011. Reenviado: 11 de noviembre de 2011. Aceptado: 15 de marzo de 2012.

Cristina Isabel Ibarra-Armenta. Es maestra en ciencias por la Universidad Autónoma de Coahuila. Actualmente cursa sus estudios de doctorado en la Universidad de Glasgow, en Glasgow, Escocia. Su línea de investigación es la economía regional y, de forma específica, la competencia territorial y la competitividad regional y de ciudades. Ha publicado, en coautoría: "A framework for assessing territorial competition: the critical role of local policy", Regional Development and policy. Challenges, choices and recipients, Memoria de Congreso, Regional Studies Association, 1720 Mayo, Newcastle Upon Tyne, (2010); "Teritorial competition in the northwest region of Mexico, 1988-2003”, Regional Responses and Global Shifts: Actors, Institutions and Organizations, Memoria de Congreso, Regional Studies Association, 24-26 mayo, Pécs, Hungari (2010).

Alejandra Berenice Trejo-Nieto. Es doctora en desarrollo por la Universidad de East Anglia. Actualmente es profesora investigadora de tiempo completo en el Centro de estudios demográficos, urbanos y ambientales de El Colegio de México. Sus líneas de investigación son la economía regional y la competitividad. Entre sus más recientes publicaciones se 
encuentran: "Territorial competitiveness in a globalised economy: regional efficiency of the mexican service sector", Chinese Business Review, 10 (4), David Publishing Company, Illinois, pp. 239-254 (2011); "The geographic concentration in the mexican manufacturing industries, an account of patterns, dynamics and explanations: 1988-2003", Investigaciones Regionales, 18, Asociación Española de Ciencia Regional, Barcelona, pp. 37-60 (2010); "The aftermath of openness and integration in the mexican manufacturing", Review of Regional Research, 30 (1), SpringerVerlag, Berlin, pp. 23-44 (2010); en coautoría, "Globalización y reestructuración económica: el caso de la industria avícola en México", en Gilberto Aboites (coord.), Patrones de consumo alimentario, Trillas, México, pp. 95-120 (2010). 